\title{
Autonomous Unmanned Ground Vehicles for Urban Logistics: Optimization of Last Mile Delivery Operations
}

\author{
Marc-Oliver Sonneberg, Max Leyerer, Agathe Kleinschmidt, Florian Knigge, Michael H. Breitner \\ Leibniz University Hannover \\ \{sonneberg; leyerer; knigge; breitner\}@iwi.uni-hannover.de \\ agathe.kleinschmidt@alumni-uni-hannover.de
}

\begin{abstract}
In an era dominated by ongoing urbanization and rising e-commerce, the efficient delivery of goods within cities becomes a major challenge. As a new element of urban logistics, we discuss the potential of autonomous unmanned ground vehicles ( $A U G V$ ) regarding the last mile delivery of shipments to customers. We propose an optimization model to minimize the delivery costs of urban shipments using AUGV. Simultaneously, best locations from a set of existing stations are selected for AUGV positioning and optimal route determination. With our developed Location Routing Problem, we provide decision support for parcel service providers, city authorities, and other relevant decision makers. Regarding the Green Information Systems domain, we tackle the lack of solution-oriented research addressing a more sustainable and locally emission free supply of goods within urban areas.
\end{abstract}

\section{Introduction and motivation}

The world's urban population is growing rapidly, already accounting for a share of 54\% [1]. Combined with the continuous growth of e-commerce, urbanization leads to increased transportation requirements in cities. This represents a challenging risk of pollution and increased traffic, influencing the health and living quality of city populations. Aspects of sustainable public transport are already tackled and implemented through subway or bus networks, as well as car- or bike-sharing. Resource-saving and sustainable business-to-consumers (B2C) transport of goods represents a growing business sector as several cities conduct pilot projects to increase sustainability.

The urban last mile delivery (LMD) is the most expensive part of the supply chain, as high personnel costs incur [2]. Consequently, transportation companies seek to improve this section of their business. City authorities are also interested in LMD because it represents a growing source of pollution. To keep cities clean and to reduce the urban road traffic, action is required. One possibility represents the delivery of goods with autonomous unmanned ground vehicles (AUGV), also referred to as delivery robots, which are subject to different restrictions compared to conventional delivery vehicles. In addition to a range limited by the battery capacity, the storage space is typically divided into compartments. This represents a considerable restriction for the use of delivery robots, which must be taken into account at route planning. The development as well as the operative use of delivery robots strongly depend on the digitalization of our society. With today's information and communication technologies, it is possible to move such robots autonomously within public space [3]. With the increasing e-commerce, more customers want to receive the ordered products as quickly as possible. This demand is addressed by same-day or even instant delivery services, where delivery robots may be able to assist the last mile transports of small goods. Based on these characteristics, delivery robots do not appear capable of solving the discussed problems completely, but represent a useful supplementary option saving personnel expenses, road space, emissions, and noise.

Besides these trends, our society is becoming increasingly aware of environmental and economic sustainability [4]. This attention is also recognized in Information System (IS) research, as information is a prerequisite for making appropriate decisions on sustainability actions [5]. The emerged research domain of Green IS addresses the transformative role of IS in the context of a sustainable society and business strategies, while considering the role of people and their livability. The foci in this field vary by conceptualization, analyses, design, and impact of such systems [5]. Studies on Green IS by Malhotra et al. [6] and Gholami et al. [7] reveal that design and impactoriented research is lacking. Since the IS domain is described as an interdisciplinary space [8], we combine elements of operations research, management science, transportation, and logistics within our approach to support locally emission free deliveries. 
Based on the situation described above, we derive our solution-oriented urban delivery approach. As part of our concept, we suggest best-possible stations from a set of existing stations, e.g., parcel service points, at strategic locations throughout the city and make use of AUGV for delivery purposes. To support the planning process of such a network consisting of stations and delivery robots for urban logistic applications, we elaborate on the following research question:

RQ: How can an IS support the establishment of an urban delivery network for route planning using autonomous unmanned ground vehicles?

To answer this question, the article is structured as follows: first, the foundations covering research design, urban logistics, autonomous unmanned vehicles, and routing problems are described. Afterwards, the optimization approach is introduced. A case study and benchmarks are presented to evaluate our approach. After a discussion regarding the delivery concept, contributions as well as limitations are elaborated. We complete our article with conclusions and outlook.

\section{Research background}

\subsection{Research design}

Our research methodology is based on Design Science Research (DSR) principles proposed by Hevner et al. [9]. This method stands in contrast to behavioral science because the design science approach systematically seeks to create "new and innovative artifacts" [10]. This means it is the most suitable approach for creating, specifying, and evaluating a particular topic while addressing its relevance and its rigor. Hevner [10] presents three cycles (relevance, rigor, and design) influencing each other. Figure 1 visualizes our design science research approach.

The topic's environment and related issues are addressed by the relevance cycle. Our research is motivated by the observation that developed AUGV are increasingly used for B2C delivery processes. This is in line with the ongoing discussion of newly and disruptive urban logistic concepts that were piloted and implemented within several projects using IS to optimize LMD activities. This trend is motivated by the continuing urbanization and the progressive climate change as air quality, traffic load, and noise pollution can be improved using delivery robots to reduce the number of trips conducted with standard delivery vehicles. By providing efficient routing support, we contribute to the lack of solution-oriented Green IS and increase the livability in cities by securing needs $[6,7]$.

Within the rigor cycle, the review of existing scientific knowledge depicts a crucial part of the research process. We carried out a literature review on the superordinate topic of urban logistics as well as on vehicle routing problems (VRP) and location routing problems (LRP). To ensure an appropriate focus on the application domain, the scope of the routing problem review is narrowed to novel applications using autonomous unmanned vehicles and their respective modeling approaches.

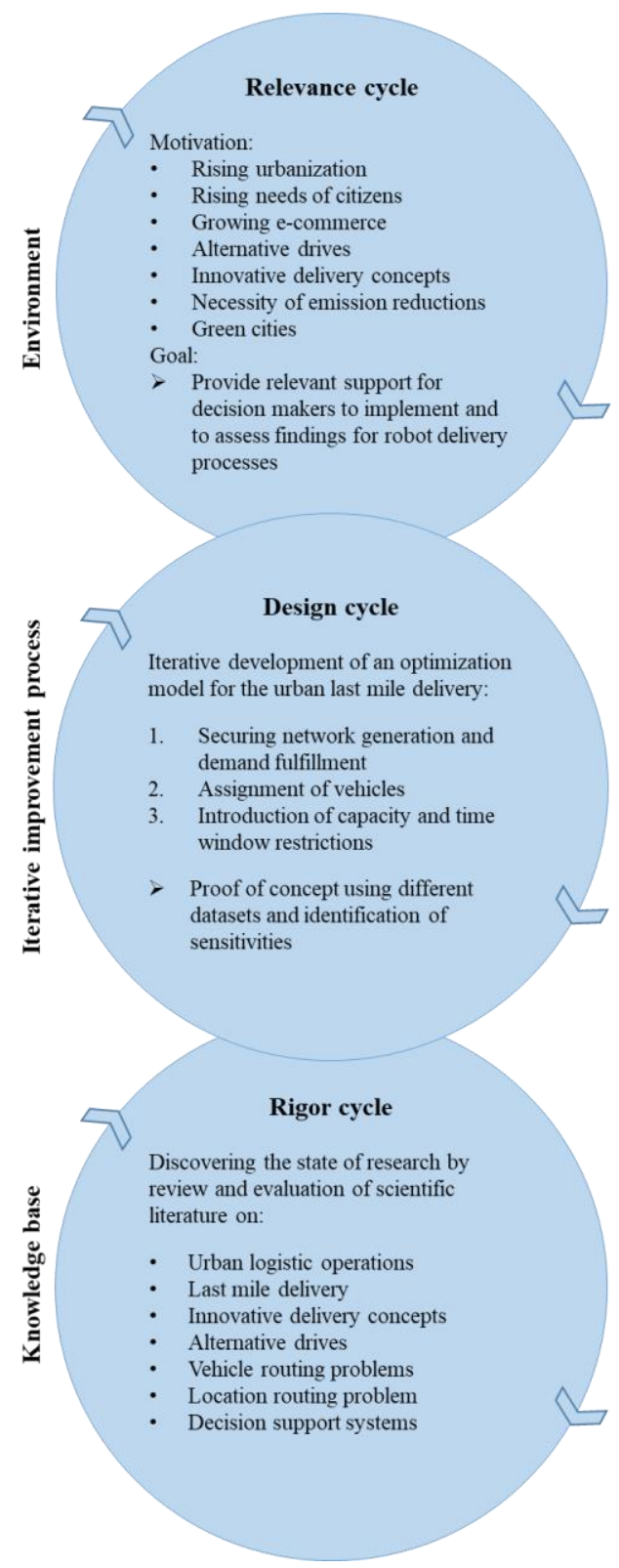

Figure 1. Applied DSR approach

The design cycle is defined as an iterative process that uses several build-and-evaluate loops and revises developed design artifacts until they are ready for a realworld application. In our research, we conducted several cycles while respecting the ongoing society trends, 
scientific methods, and existing knowledge. Our approach covers a concept for the urban LMD of goods by using AUGV with an effective network of stations within urban areas. The mathematical model was iteratively developed, verified, and improved at each stage of the design process to allow for relevant decision support. By conducting several benchmark calculations, we tested the optimization model while obtaining sensitivities of the results to enable proper documentation of results.

\subsection{Urban logistics}

The term urban logistics is defined as "the movement of goods, equipment and waste into, out, from, within or through an urban area" [11]. Closely related to this definition is the term city logistics that is specified as "process for totally optimising the logistics and transport activities by private companies with support of advanced information systems in urban areas considering the traffic environment, the traffic congestion, the traffic safety and the energy savings within the framework of a market economy" [12]. As already mentioned above, the trends of a growing ecommerce and urbanization lead to an increasing number of transport activities in cities. Concurrently politics and city authorities aim for eco-friendly logistic solutions in the future, which likely become challenging for the transport sector. When addressing the subject of sustainability, the considerate handling of given resources becomes a crucial aspect.

To make our approach most relevant for practical urban applications, this article concentrates on the optimization of the LMD. Within the (global) supply chain of goods, LMD represents the final and most expensive section of the transport chain [2]. Today, trucks and light duty vehicles perform the majority of urban logistic activities. Different strategies and concepts aim to improve the LMD and the city logistics in general regarding environmental issues. Besides alternative transport vehicles (e.g., electric cars or cargo bicycles), the use of urban distribution centers is a feasible approach to implement new elements to the city infrastructure [13, 14]. However, further innovative forms of operations are required to achieve an integrated city structure [15]. Intelligent transport systems can contribute to increase the efficiency of LMD activities [16]. Several ongoing projects investigate sustainable LMD solutions such as pro-e-bike, cyclelogistics, Civitas, FREVUE, and many others. These projects focus on sharing best practices between different cities or on testing innovative distribution concepts to introduce eco-friendly ways of urban freight transportation. Most of these concepts support parcel service providers to implement improved logistic concepts and encourage manufacturers to offer ecofriendly transport vehicles to reach a persistent change regarding a more sustainable way of urban life.

\subsection{Autonomous unmanned vehicles}

In the last decade, autonomous unmanned vehicles have been designed and used for various applications, e.g., for container handling at port operations, intra- as well as hospital and medicine logistics [17]. Nowadays, autonomous unmanned vehicles are also developed to assist B2C logistic operations within public spaces, whereas they can be differentiated between aerial- and ground-operated vehicles. Autonomous unmanned aerial vehicles (AUAV), also referred to as drones, and AUGV were especially tested within pilot projects to investigate the feasibility as well as customer acceptance. In our approach, we focus on AUGV in the public space using so called delivery robots with the goal of transporting small shipments to private customers. Due to their size, they are designed for the use on pedestrian walkways, but generally not for traffic on public roads. Compared to traditional delivery vehicles such as trucks, vans, or cars, delivery robots can only transport a few shipments. The number of shipments per tour and per AUGV depends on the number of individually lockable compartments. These compartments, which are electronically secured against unauthorized access, can be unlocked at the destination by the recipient with an individual code, so that each recipient only has access to his own shipment. Delivery robots run electrically and are therefore limited in terms of range. Thus, they are particularly suitable for use in the last mile for transporting shipments over short distances. On their way to the customers, the vehicles avoid obstacles autonomously. The length of the route is affected by the number of obstacles a robot encounters on its tour to the customer's door. Obstacles can be groups of people, stairs, secured properties, or other objects that robots cannot overcome. In situations where a robot cannot find a way out, a so-called operator can take over the control, who has access to cameras and other sensors of the AUGV via the mobile network.

In practice, a rather small number of delivery robot manufacturers exists. The most popular providers are Starship Technologies, Dispatch, and Marble. These three companies are currently carrying out various pilot projects in several cities, whereas many of the other manufacturers are still in the development phase not operating in public space yet. In various projects, Starship Technologies even tested the supply of prepared dishes, e-grocery, or the dispatch of medicines [18]. First analyses show that the public's perception of the delivery with autonomous vehicles is mostly positive and predict an urban parcel delivery rate of up 
to 80 percent by autonomous vehicles in 2025 accounting for cost advantages of parcel service providers up to 40 percent [19].

\subsection{Vehicle routing problems}

In view of the large body of scientific works, the VRP is widely considered as a distinct field of knowledge in operations research and computer science $[20,21]$. Regarding the planning horizon of transport and logistics processes, the VRP assists short-term and daily decisions related to diverse transportation services of goods and passengers. The LRP can be understood as a special case of the VRP. In addition to route optimization, the ideal number of locations (e.g., depots, warehouses, stations, etc.) is determined simultaneously. In doing so, LRP approaches focus on the operational and the tactical decision level. First route planning analyses have been carried out for more than 50 years by Danzig and Ramser [22]. Yet, this section aims to give a brief overview about VRP and LRP focused on autonomous techniques, which is an emerging field of route planning.

The first published VRP concerning autonomous vehicles were focused on the application of logistic port operations. The central optimization objective was mainly the minimization of makespans or costs for efficient unmanned container scheduling. Examples for such operations are Geraleh et al. [23], Xin et al. [24], or Schmidt et al. [25]. Further VRP applications are developed for intra-logistic operations minimizing makespans or penalizing earliness and tardiness, as for instance presented by Dang and Nguyen [26] or by Fazlollahtabar et al. [27]. In 2015, Murray and Chu [28] present an article concerning AUAV and different operation modes minimizing the aerial delivery time. Other optimization approaches regarding urban LMD also apply AUAV. Shavarani et al. [29] introduce a facility location problem to determine optimal number and locations of launch and recharge stations for AUAV minimizing overall system costs. However, most of existing approaches combine a classical parcel delivery with trucks together with AUAV services (also as referred to as tandem delivery) to maximize the total delivery efficiency [30, 31, 32, 33]. Similarly, Boysen et al. [34] present an optimization model for combining trucks and AUGV together for minimizing the weighted number of late deliveries without considering cost components. There are also approaches regarding the optimization and planning of healthcare and hospital logistics [35, 36] or automated cleaning services [37]. To conclude, certain articles deal with autonomous VRP and its diverse specifications on different operational purposes. To our knowledge, no research on VRP and especially LRP concerning the sole application of
AUGV for urban logistic applications is already existing. To address this lack, we focus on these operations with the objective of minimizing delivery costs. For proper development of our optimization model presented in the next section, we take ideas, considerations, and assumptions of the above mentioned articles into account.

\section{Optimization approach}

\subsection{Problem, assumptions, and notation}

This section introduces a mixed-integer linear problem for optimizing LMD operations of AUGV. This is characterized as a special case of the LRP, related to the use of electrically driven delivery robots under consideration of demand- and supply-related time windows. These robots start and end their tours at one of several potential stations (e.g., at parcel service points) and visit customer locations to supply them with their demanded goods.

By solving the model with the goal of minimizing total costs, simultaneously the number and locations of the stations to be opened, the assignment of customers to these stations, the number of necessary AUGV, and the number as well as the routes of the driving tours are determined. Within the framework of route planning, the customers are grouped into tours and the sequence for delivery to customers within the tours is optimized. In addition to time restrictions for delivery, capacity restrictions regarding the battery and the transport volume of the delivery robots are considered. Summing up, the model is classified as electric location routing problem with time-windows (ELRP-TW). To allow for the optimization of the underlying problem, the following assumptions must be made:

- The potential locations of the stations are given.

- There is sufficient space at the potential stations for short-term storage of shipments ready for dispatch as well as for the charging infrastructure of the delivery robots.

- Since each AUGV is assigned to a station, each tour starts and ends at the same place.

- The locations as well as the associated demand levels of the customers are given.

- Maximum possible transport weight as well as volume are considered and the capacity restrictions are met. The individual orders are referred to as a uniform package, so that each package unit represents the maximum weight and volume of the delivery robot. The demand therefore represents a certain number of uniform packages.

- Identical AUGV with a limited transport capacity are used for shipment delivery whereby each 
delivery robot has a certain number of separated compartments for serving different customers. A delivery robot's compartment can contain one uniform package.

- Each delivery robot is able to conduct several tours per day by taking capacity limits into account. Sufficient time to prepare the AUGV for its delivery between two consecutive tours exists, and, if necessary, to change the battery.

- Each customer location is served by at least one vehicle and split deliveries are possible.

- Since shipments are delivered within time windows, the demand can be fully met so that undeliverable shipments and multiple delivery attempts do not exist. As a result, each customer is available to receive his shipment at the desired time window.

The indices, parameters, and decision variables are summarized in the following:

\begin{tabular}{|c|c|}
\hline$g, h \in \mathcal{G}$ & Set of locations / graph nodes \\
\hline$i, l \in \mathcal{J} \subseteq \mathcal{G}$ & Set of customers demand locations \\
\hline$j \in \mathcal{J} \subseteq \mathcal{G}$ & Set of potential stations \\
\hline$k, o \in \mathcal{K}$ & Set of tours \\
\hline$r \in \mathcal{R}$ & Set of potential delivery robots \\
\hline$a_{i}$ & Demand of customer $i$ \\
\hline$b^{\text {range }}$ & Maximum battery electric range of a delivery robot \\
\hline$b^{\text {time }}$ & $\begin{array}{l}\text { Maximum battery operating time of a delivery } \\
\text { robot }\end{array}$ \\
\hline$c$ & Transport costs per distance unit \\
\hline$d_{g h}$ & Distance between location $g$ and $h$ \\
\hline$e$ & Auxiliary parameter \\
\hline$f$ & Daily rental fee per delivery robot \\
\hline$M$ & Sufficiently large number \\
\hline$n$ & Number of minutes of a time interval \\
\hline$p$ & Personnel costs per minute \\
\hline$q_{j}^{\text {station }}$ & Storage capacity of station $j$ \\
\hline$q^{\text {robot }}$ & Number of a delivery robot's compartments \\
\hline$s^{\text {range }}$ & Safety buffer for the battery electric range \\
\hline$s^{\text {time }}$ & Safety buffer for the battery operating time \\
\hline$t^{\text {load }}$ & Loading time per shipment \\
\hline$t^{\text {serv }}$ & Service time per customer \\
\hline$t_{g h}^{t i m e}$ & Travel time from location $i$ to $g$ \\
\hline$v_{j}^{\text {close }}$ & Closing time of station $j$ \\
\hline$v_{j}^{\text {open }}$ & Opening time of station $j$ \\
\hline$w_{i}^{\text {close }}$ & Latest possible delivery time of customer $i$ \\
\hline$w_{i}^{\text {open }}$ & Earliest possible delivery time of customer $i$ \\
\hline$\alpha_{g h k}$ & $\begin{array}{l}\text { 1, if tour } k \text { leads from location } g \text { to location } h \\
0, \text { else }\end{array}$ \\
\hline$\beta_{r j}$ & $\begin{array}{l}1 \text {, if robot } r \text { is located at station } j \\
0 \text {, else }\end{array}$ \\
\hline$\gamma_{j}$ & $\begin{array}{l}1 \text {, if a station is selected at location } j \\
0 \text {, else }\end{array}$ \\
\hline$\delta_{g k}$ & Arrival time of tour $k$ at location $g$ \\
\hline$\varepsilon_{i j k}$ & Compartments of station $j$ in tour $k$ to customer $i$ \\
\hline$\zeta_{k r}$ & $\begin{array}{l}1 \text {, if tour } k \text { is driven by robot } r \\
0, \text { else }\end{array}$ \\
\hline
\end{tabular}

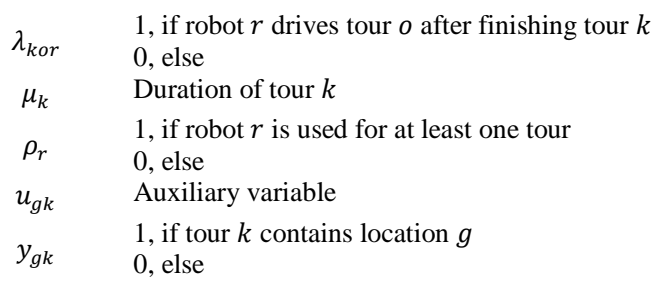

\subsection{Mathematical model}

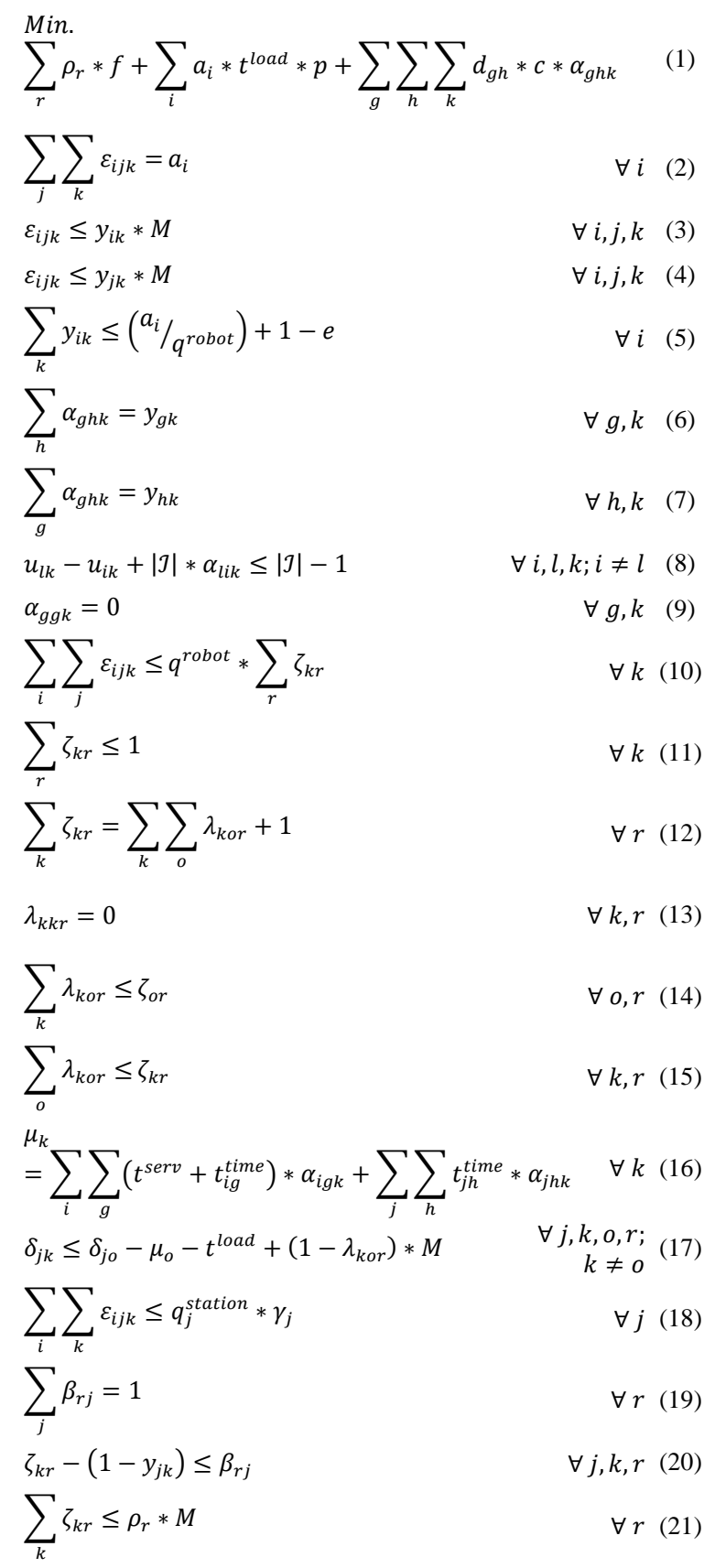




$$
\begin{array}{lr}
\sum_{g} \sum_{h} d_{g h} * \alpha_{g h k} \leq b^{\text {range }}-s^{\text {range }} & \forall k(22) \\
\mu_{k} \leq b^{\text {time }}-s^{\text {time }} & \forall k(23) \\
\delta_{i k} \geq w_{i}^{\text {open }} & \forall i, k(24) \\
\delta_{i k} \leq w_{i}^{\text {close }} & \forall i, k(25) \\
\delta_{g k} \geq \delta_{i k}+t^{\text {serv }}+t_{i g}^{\text {time }}-\left(1-\alpha_{i g k}\right) * M & \forall g, i, k(26) \\
\delta_{g k} \leq \delta_{i k}+t^{\text {serv }}+t_{i g}^{\text {time }}-\left(1-\alpha_{i g k}\right) * M & \forall g, i, k(27) \\
v_{j}^{\text {open }}+t_{j i}^{\text {time }}-\delta_{i k}-\left(1-\alpha_{i g k}\right) * M \leq 0 & \forall i, j, k(28) \\
\delta_{j k} \leq v_{j}^{\text {close }} & \forall j, k(29) \\
\alpha_{g h k}, \beta_{r j}, \gamma_{j}, \zeta_{k r}, \lambda_{k o r}, \rho_{r}, y_{g k} \in\{0,1\} & \forall g, h, i, j, k, o, r(30) \\
\delta_{g k}, \mu_{k}, u_{g k} \geq 0 & \forall g, k(31) \\
\varepsilon_{i j k} \in \mathbb{Z}_{\geq 0} & \forall i, j, k(32)
\end{array}
$$

The objective function (1) minimizes the total costs of one working day. These consist of the rental costs of all utilized delivery robots, the personnel costs for preparing the robots, and the variable delivery costs for all tours. The latter correspond to the total distance to be covered multiplied with the transport cost rate $c$. As it is assumed that the stations are placed at existing locations with an existing workforce, the personnel costs are modelled according to the time that is needed to prepare the robots for delivery purposes.

Constraints (2) to (9) refer to the classical VRP. Constraint (2) requires that the entire order quantity is delivered to the corresponding customer. Constraint (3) ensures that a customer $i$ is only supplied within tour $k$ if this customer is assigned to the tour $k$ using the binary variable $y_{i k}$. Similarly, condition (4) assigns the stations $j$ to the tours $k$. Using the auxiliary parameter $e$, constraint (5) ensures that a customer is not visited more often than necessary. The maximum number of deliveries is determined based on the order quantity and robot capacity. Constraint (6) guarantees that on tour $k$ the location $h$ can only be reached from location $g$ if this place $g$ is also included in tour $k$. Similarly, constraint (7) requires that tour $k$ leads from location $g$ to location $h$ only if that tour also includes the location $h$. Constraint (8) serves to avoid short cycles which are tours that do not include a station. The auxiliary variable $u_{i}$ assumes a higher value the later the location $l$ is visited in tour $k$. Further, constraint (8) secures that a tour cannot end in a customer location. Constraint (9) causes a location not to be accessed by itself.

Constraints (10) to (17) exist for the assignment of AUGV to tours. Constraint (10) ensures that deliveries are only made during tour $k$ if a robot is assigned to this tour, and furthermore that the delivery quantity does not exceed the AUGV's capacity. Due to constraint (11) only one AUGV can be assigned to a tour. Constraint (12) implies that a sufficient number of binary variables $\lambda_{\text {kor }}$ takes the value 1 to represent the tour order driven by robot $r$. Constraint (13) ensures that in the case of a robot with only one assigned tour, the variable $\lambda_{\text {kor }}$ is zero for all $k$ and all $m$. Constraint (14) requires that the adjacent tour $m$ after the following tour $k$ can only be scheduled for robot $r$ if tour $m$ is assigned to this robot. Similarly, the constraint (15) ensures that tour $k$ can only be scheduled before tour $m$ of robot $r$ if the tour $k$ is assigned to this robot. Using constraint (16), the variable $\mu_{k}$ is assigned to a value equal to the time required for tour $k$. If robot $r$ drives tour $m$ directly after tour $k$, the constraint (17) requires that these tours do not overlap in time and that there is sufficient time between them for replacing the battery as well as loading the shipments into the AUGV.

The constraints (18) to (21) control the assignment of AUGV's to stations. Constraint (18) requires that customer $i$ can only be supplied from location $j$ if a station is selected there. Furthermore, this constraint prevents the available capacity of a station from being exceeded by the cumulative demand of the customer locations assigned to it. Constraint (19) requires that each robot is positioned at exactly one station. Furthermore, restriction (20) ensures that a robot is only assigned to tours that begin at its associated stations. Constraint (21) secures that each utilized robot must be assigned to at least one tour.

Constraint (22) ensures that no tours are planned that exceed the electric range of the battery. The safety buffer on the right side of the inequality assures that a certain reserve for unforeseen events (e.g., impassable pathways) is held back during the tour. Similarly, constraint (23) adds a time-dependent safety buffer for a tour to not exceed the maximum usage time of the battery.

The auxiliary conditions (24) to (29) refer to the observance of time windows in the context of tour planning and delivery processes. For this, constraint (24) requires that a customer location is not reached before its earliest possible delivery time. Similarly, constraint (25) prevents a customer location from being visited after the corresponding time window has expired. Constraint (26) ensures that within tour $k$ the arrival time at location $g$ is not before the arrival time at location $i$ plus the service time and travel time between both locations. Similarly, constraint (27) requires that within tour $k$ the arrival time at location $g$ is not after the arrival time at location $i$ plus service time and travel time between both locations. Constraint (28) causes that the first customer location $i$ of a tour cannot be supplied before opening the assigned base station $j$ plus the respective travel time. For each station $j$, constraint (29) secures that the arrival time of tour $k$ must not be after the end of the delivery time. The constraints (30), (31), and (32) limit the permissible value range of the decision variables. 


\section{Proof of concept}

\subsection{Single case study}

The described optimization model is developed to establish a delivery network of stations to determine the optimal assignment of customers to these stations. The amount of AUGV as well as the number and the customer order of the driving tours are calculated as well. When applying the optimization approach, the quality and level of the input values strongly affect the results of the underlying model. This not only includes cost-related parameters, such as vehicle and personnel costs, but also the assumed demand, which considerably influences the solution.

In the following case study, three potential stations are available to be opened. At least one of them has to fulfill the requests of ten demand locations. These demand locations are assigned to at least one selected station. The locations are distributed within an area of $3000 \times 3000$ meters (m) and can therefore be used to determine the distances and resulting driving time. One time interval is equal to one hour and contains 60 minutes. The storage capacity of a station $\left(q_{j}^{\text {station }}\right)$ is set to fifty shipments, as the place within a parcel service point is limited. These service points are open from 8:00 to 20:00. Based on the number of compartments, an AUGV is able to deliver several shipments simultaneously. The following Table 1 summarizes the parameter values applied within this example.

Table 1. Assigned parameter values

\begin{tabular}{|c|c|c|c|}
\hline Parameter & Value [unit] & Parameter & Value [unit] \\
\hline$b^{\text {range }}$ & $10,000[\mathrm{~m}]$ & $p$ & $0.5[€ / \mathrm{min}]$ \\
\hline$b^{\text {time }}$ & $360[\mathrm{~min}]$ & $s^{\text {range }}$ & $200[\mathrm{~m}]$ \\
\hline$c$ & $0.001[€ / \mathrm{m}]$ & $s^{\text {time }}$ & $36[\mathrm{~min}]$ \\
\hline$f$ & $20[€ /$ day $]$ & $t^{\text {load }}$ & $1[\mathrm{~min}]$ \\
\hline$M / \mathrm{e}$ & $10000 / 0.0001$ & $t^{\text {serv }}$ & $10[\mathrm{~min}]$ \\
\hline
\end{tabular}

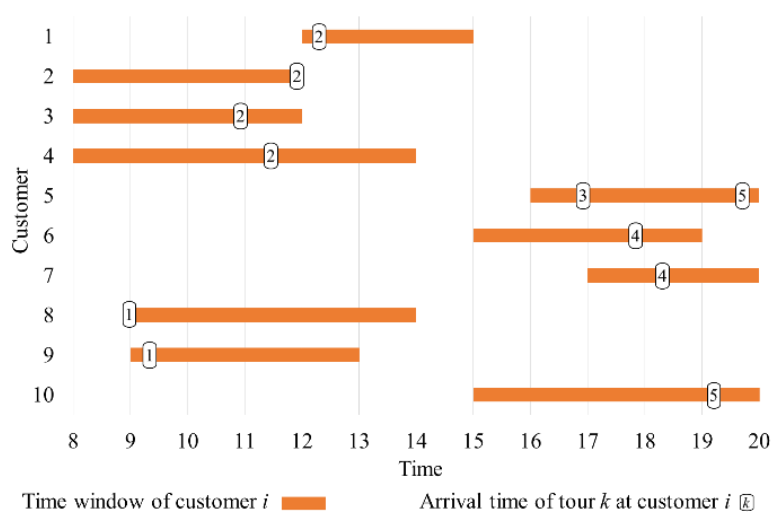

Figure 2. Customers' time windows and arrival times
The demand level per customer varies between one and six shipments resulting in an aggregated demand of 20 shipments. The requested time windows of the customers of this example are shown in Figure 2. The optimized arrival times of the shipments are also visualized. For this case study, the number of compartments per AUGV ( $q^{\text {robot }}$ ) is set to four.

The total costs, represented by the objective function value (OFV), are $45.82 € /$ day and consist of $20 € \mathrm{AUGV}$ rental costs (43.65\%), $10 €$ personnel costs for preparing the robot (21.82\%), and $15.82 €$ variable distance costs $(34.53 \%)$. As a result, one station is opened. Starting from this, five tours are driven by one AUGV. To satisfy all customer demands within their specified time window, one battery swap is necessary.

The calculation for this application case and the following benchmarks were performed on a standard computer (Intel Core i5-6200 CPU, $2.30 \mathrm{GHz}$, and $8 \mathrm{~GB}$ RAM) using modelling software GAMS 24.5.6 with the solver CPLEX. We limit the computation time to 10,000 seconds and an optimization gap of 5\%.

\subsection{Benchmarks on AUGV compartments}

To show the influences on the total driving distance as well as on the OFV, we provide benchmarks varying the number of compartments per AUGV $\left(q^{\text {robot }}\right)$ ceteris paribus. The following Figure 3 visualizes these results. The total daily costs (blue font) are shown on the left vertical axis and the resulting distance covered (green font) is indicated on the right vertical axis.

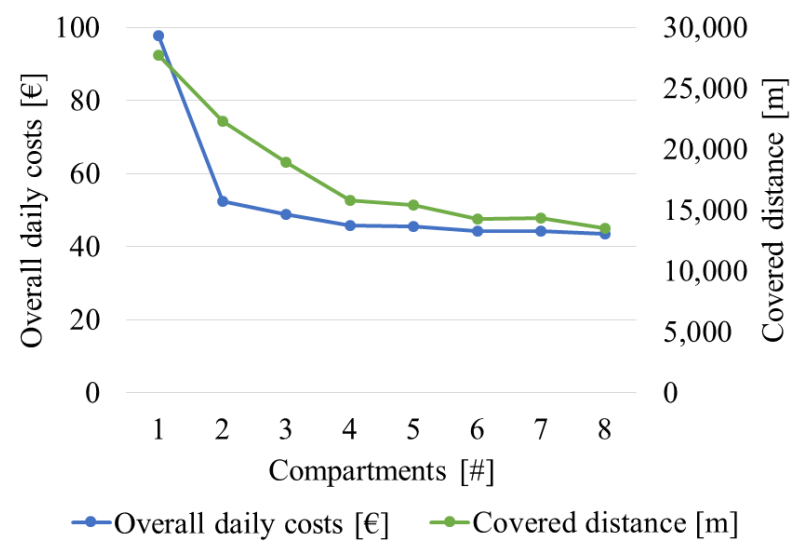

\section{Figure 3. AUGV compartment benchmarks}

It is apparent that if the number of compartments increases, both the total costs and the distance travelled decrease. The total daily costs are nearly halved (- 46.45\%) when two compartments are present instead of one. This is because three AUGV are required for the delivery of the 20 shipments due to time window restrictions as each shipment is carried alone. When installing two or more compartments, it is sufficient to 
use only one AUGV. For this reason, the financial savings of two compartments compared to eight compartments are rather low. These financial differences are based on lower variable vehicle costs as the covered distance decreases. In the one compartment case, each customer is visited once per shipment, even if a customer demands more than one shipment. This way of delivery can be described as round-trip delivery, whereby pooling advantages due to the proximity of different customers are not possible. These benefits can increase the more customers are supplied on a tour. This combination allows to gradually reduce the distance covered. Up to a compartment number of four, the covered distance decreases significantly, further rangerelated advantages are rather less cost-efficient.

As shown, the number of compartments depict a crucial parameter for route planning with AUGV. Depending on the purpose and size of the shipments, the use of AUGV with multiple compartments is beneficial as several customers can be supplied within one tour. The use of an AUGV with only one compartment is less cost-efficient, also if two of three manufacturers of AUGV for delivery purposes offer a single compartment robot. To conclude, the operational effectiveness depends on the present distances and the (size and weight of the) transported goods (e.g., prepared dishes, e-grocery, or the dispatch of medicines).

\section{Discussion and implications}

We answer our research question with the developed ELRP-TW as final artifact of the applied DSR process. Our ELRP-TW represents a special case of the LRP for the application of AUGV in the context of urban parcel delivery depicting a new element for urban logistics operations. By solving the model with the main goal of minimizing the total daily costs, we are able to select optimal locations from a set of existing stations and to determine the quantity of $\mathrm{AUGV}$ and its tours simultaneously. Restrictions regarding the battery capacity and time windows for delivery to customers were considered.

First of all, the possibilities for implementing the delivery concept within urban spaces must be mentioned. Based on the assumption that only AUGV are used to supply customers, the ELRP-TW is unsuitable for some business models and application areas. Especially when the expected order quantities vary considerably in terms of volume as well as weight, the AUGV's capacity can be exceeded. In such cases, a combination of AUGV and conventional delivery vehicles, such as vans, is plausible as suggested by [34]. However, the shipments to be delivered must be preselected in terms of volume to assign the small-sized packages to AUGV.
Not to be ignored is also the field of application as urban areas are differently designed. Since the AUGV move exclusively on footpaths, it is possible that these paths are inappropriate or that too many pedestrians hinder an efficient movement. Further, in some countries, there are also strict legal restrictions that prevent the operation of AUGV in public spaces. However, forecasts on automated unmanned vehicles within the urban delivery as well as investigations regarding customer acceptance reveal positive future projections for this field [19].

A crucial factor regarding the delivery robots' efficiency is the number of its compartments. It is notable that the Starship robot and the Marble robot only have one single lockable compartment, whereas Dispatch offers a robot with four compartments. As consequence, the Starship and Marble robots can only supply one customer per tour, so that each tour consists of a round-trip with a single destination. The Dispatch robot, on the other hand, can supply up to four customers per tour so that the routes can be optimized across all tours. Yet, these differences also result in a variety of possible applications. The conducted sensitivity analysis indicates a strong relationship between robot capacity and total costs. As increasing the robot's number of compartments lowers the resulting overall costs, the further development of delivery robots with multiple compartments makes sense.

\section{Contributions}

Our approach integrates existing knowledge on VRP and LRP together with computer-aided decision support while combining scientific literature of transportation, logistics, operations research as well as management science. Previous research activities in these fields demonstrate the lack of decision support for the urban delivery with AUGV and the related route planning and optimization. In doing so, we promote innovative delivery concepts and their manifold implementation possibilities. With the developed optimization model, we assist an efficient implementation of the proposed logistics concept.

We further contribute to the Green IS literature as our research addresses relevant issues regarding the locally emission free supply of goods within urban areas. We developed an optimization model which assists the planning of the urban LMD by using AUGV. With our approach, we reacted to the call of Malhotra et al. [6] and Gholami et al. [7], who point out the overrepresentation of conceptualization and analyses compared to solution-oriented research. We combined transportation and Green IS research to promote the transformative role of IS in contributing to enhanced economic and environmental sustainability. With the 
help of the optimization model, we enable decisionmaking for parcel service providers, city authorities, and other (potential) stakeholders (e.g., provider of prepared meals) in finding appropriate solutions for the urban delivery of goods with AUGV. By using this concept, it is possible to optimize the last mile and thereby to reduce personnel costs as well as pollution, road space, and noise to increase the livability in urban areas.

\section{Limitations}

The model formulation of the ELRP-TW aims at the simultaneous determination of station locations, AUGV quantity, and tours. As a result, this optimization model can only be used for applications where location planning is carried out at the same frequency as route planning. One example of this could be the daily repositioning of mobile depots in the form of trucks or containers from which AUGV carry out deliveries on the last mile. However, it is possible to fixate the selected stations for following optimizations resulting in an adjusted VRP. Further, the model could be used for network expansion reasons raising the number of stations to meet increased demands.

In any case, the model-user must be aware that the optimization problem can become infeasible, if a customer is too far away from any potential station. Also, too narrow time windows can lead to incompatibilities. It should be noted that the actual weight and the dimension of the shipments are not modelled. In reality, shipments differ in a wide range.

As the ELRP-TW is based on the LRP which is classified as np-hard problem. Since the computing effort increases exponentially with increasing problem size, optimal or sufficiently good solutions for large problem instances cannot typically be found within reasonable computing time [38]. Regarding the results already discussed, it should be noted that all calculations in the context of this work were based on a small problem instance with fictive demand levels. Based on the input parameters, an optimization process can take several hours. For a more differentiated assessment, further analyses using larger problem instances are recommended.

In addition, Euclidean distance measurement (L2 metric) is used as basis for the application case. However, this distance measurement is only limitedly suitable for practical use in real applications in urban logistics. Instead, it is preferable to use the actual distances of the routes envisaged. For example, data could be obtained from web-based routing providers offering application programming interfaces.

To conclude, the assumptions, the optimization model, and the input parameters serve as starting points for future research regarding the topic of AUGV within urban delivery operations. It should be noted that the utilization of delivery robots cannot solve the problems of urban logistics on its own but can make a beneficial impact in taking over certain consignments. They could also be responsible for the short-term dispatch of locally available goods to the end customer, such as same-day or same-hour deliveries.

\section{Conclusions and outlook}

The ELRP-TW was presented as an optimization model for simultaneous planning of locations, delivery robots, and tours to maximize the AUGV application efficiency. Due to increasing urbanization worldwide, this integration of a new element within urban logistics represents a promising approach for various delivery operations reducing personnel costs, emissions, road space, and noise for more sustainable and locally emission free deliveries. However, it should be noted that the use of AUGV cannot fully compensate existing delivery operations. Yet, AUGV will cause a perceptible impact taking over special shipments promoting new business models for urban delivery, e.g., same-day or same-hour deliveries. We recommend future research on AUGV applications in the field of urban logistics and the implementation of our implications for better planning, optimization, and decision support.

\section{Acknowledgements}

This work is part of the joint research project "USEfUL" (fund number 03SF0547) funded by the German Federal Ministry of Education and Research $(\mathrm{BMBF})$ and managed by the Project Management Jülich.

\section{References}

[1] United Nations, World Urbanization Prospects: The 2014 Revision, Department of Economic and Social Affairs, NY: New York, 2014.

[2] R. Gevaers, E. van de Voorde, and T. Vanelslander, "Characteristics of Innovations in Last-mile Logistics - Using Best Practices, Case Studies and Making the Link with Green and Sustainable Logistics", Department of Transport and Regional Economics, University of Antwerp, 2009, 21 p.

[3] U. Günter, Automated Guided Vehicle Systems: a Primer with Practical Applications, 2. rev. and expanded ed., Springer, Berlin, 2015.

[4] J. Dedrick, "Green IS: Concepts and Issues for Information Systems Research", Communications of the Association for Information Systems (27), 2010, pp. 173-184.

[5] R. T. Watson, M. C. Boudreau, and A. J. Chen, "Information Systems and Environmentally Sustainable Development: Energy Informatics and New Directions for the 
IS Community", Management Information Systems Quarterly (34:1), 2010, pp. 23-38.

[6] A. Malhotra, N. P. Melville, and R. T. Watson, "Spurring Impactful Research on Information Systems for Environmental Sustainability", Management Information Systems Quarterly (37:4), 2013, pp. 1265-1274.

[7] R. Gholami, R., R. T. Watson, A. Molla, H. Hasan, and N. Bjørn-Andersen, "Information Systems Solutions for Environmental Sustainability: How Can We Do More?“, Journal of the Association for Information Systems (17:8), 2016, pp. 521-536.

[8] N. R. Hassan, "Value of IS Research: Is there a Crisis?", Communications of the Association for Information Systems (34:41), 2014, pp. 801-816.

[9] A. R. Hevner, S. T. March, J. Park, and S. Ram, "Design Science in Information Systems Research", Management Information System Quarterly (28:1), 2004, pp. 75-105.

[10] A. R. Hevner, "A Three Cycle View of Design Science Research", Scandinavian Journal of Information Systems (19:2), 2007, pp. 87-92.

[11] European Commission, A Call to Action on Urban Logistics, Commission Staff Working Document, BE: Brussels, 2013.

[12] E. Taniguchi, R. G. Thompson, T. Yamada, and R. van Duin, "City Logistics: Network Modelling and Intelligent Transport Systems", Pergamon, UK: Oxford, 2001.

[13] M. G. Speranza, "Trends in Transportation and Logistics", European Journal of Operational Research (264), 2018, pp. 830-836.

[14] M. Savelsbergh, and T. Van Woensel, "City Logistics: Challenges and Opportunities", Transportation Science (50:2), 2016, pp. 579-590.

[15] United Nations, Planning and Design for Sustainable Urban Mobility: Global Report on Human Settlements, Routledge, UK: Abingdon, 2013.

[16] E. Taniguchi, "Concepts of City Logistics for Sustainable and Livable Cities", Procedia - Social and Behavioral Sciences (151), 2014, pp. 310-317.

[17] A. Finn, and S. Scheding, "Developments and Challenges for Autonomous Unmanned Vehicles", Intelligent Systems Reference Library (3), 2010, pp. 128-154.

[18] Starship, https://www.starship.xyz/business, 2017 (Accessed: 2018/08/31).

[19] McKinsey, Parcel delivery - The Future of Last Mile, Travel, Transport and Logistics, 2016.

[20] R. Baldacci, M. Battarra, D. and Vigo, "Routing a Heterogeneous Fleet of Vehicles", in: The Vehicle Routing Problem: Latest Advances and New Challenges, Springer, Boston, 2018.

[21] P. Toth, P. and D. Vigo, "The Vehicle Routing Problem, Philadelphia, PA: Society for Industrial and Applied Mathematics, 2001.

[22] G. B. Dantzig and J. H. Ramser, "The Truck Dispatching Problem", Management Science (6:1), 1959, pp. 80-91.

[23] S. Gelareh, R. Merzouki, K. McGinley, and R. Murray, "Scheduling of Intelligent and Autonomous Vehicles under Pairing/Unpairing Collaboration Strategy in Container Terminals", Transportation (33:1), 2013, pp. 1-21.

[24] J. Xin, R. R. Negenborn, F. Corman, and G. Lodewijks, "Control of Interacting Machines in Automated Container Terminals using a Sequential Planning Approach for Collision
Avoidance", Transportation Research Part C: Emerging Technologies (60), 2015, pp. 377-396.

[25] J. Schmidt, C. Meyer-Barlag, M. Eisel, L. M. Kolbe, and H. J. Appelrath, "Using Battery-Electric AGVs in Container Terminals: Assessing the Potential and Optimizing the Economic Viability", Research in Transportation Business \& Management (17), 2015, pp. 99-111.

[26] Q.V. Dang and L. Nguyen, "A Heuristic Approach to Schedule Mobile Robots in Flexible Manufacturing Environments", Proceedings 40th CIRP, 2016, pp. 390-395.

[27] H. Fazlollahtabar, M. Saidi-Mehrabad, and J. Balakrishnan, "Mathematical Optimization for Earliness / Tardiness Minimization in a Multiple Automated Guided Vehicle Manufacturing System via Integrated Heuristic Algorithms", Robotics and Automated Systems (72), 2015, pp. 131-138.

[28] C. C. Murray and A. G. Chu, "The Flying Sidekick Traveling Salesman Problem: Optimization of Drone-Assisted Parcel Delivery", Transportation Research Part C: Emerging Technologies (54), 2015, pp. 86-109.

[29] S. M. Shavarani, M. G. Nejad, F. Rismanchian, and G. Izbirak, "Application of Hierarchical Facility Location Problem for Optimization of a Drone Delivery System: a Case Study of Amazon Prime Air in the City of San Francisco", International Journal of Advanced Manufacturing Technology (95:9-12), 2015, pp. 3141-3153.

[30] H. H. Minh, Y. Deville, Q. D. Pham, and M. H. Quang, "Heuristic Methods for the Traveling Salesman Problem with Drone", ICTEAM/INGI/EPL report, 2015, 13 p.

[31] N. Agatz, P. Bouman, and M. Schmidt, "Optimization Approaches for the Traveling Salesman Problem with Drone", Transportation Science (52:4), 2018, pp. 965-981.

[32] J. G. Carlsson, and S. Song, "Coordinated Logistics with a Truck and a Drone", Management Science (64:9), 2017, pp. 4052-4069.

[33] S. M. Ferrandez, T. Harbison, T. Weber, R. Sturges, and R. Rich, "Optimization of a Truck-Drone in Tandem Delivery Network using K-Means and Genetic Algorithm", Journal of Industrial Engineering and Management (9:2), 2016, pp. 374388.

[34] N. Boysen, S. Schwerdfeger, and F. Weidinger, "Scheduling Last-Mile Deliveries with Truck-based Autonomous Robots", European Journal of Operational Research (271:3), 2018, pp. 1085-1099.

[35] J. Bačík, F. Durovský, M. Biroš, K. Kyslan, D. Perduková, and S. Padmanaban, "Pathfinder-Development of Automated Guided Vehicle for Hospital Logistics", IEEE Access (5), 2015, pp. 26892-26900.

[36] J. Scott, and C. Scott, "Drone Delivery Models for Healthcare", Proceedings of the 50th Hawaii International Conference on System Sciences, 2017, 8 p.

[37] S. Jeon, M. Jang, D. Lee, Y. J. Cho, J. Kim, and J. Lee, "Multiple Robots Task Allocation for Cleaning and Delivery", in: Intelligent Systems and Applications, Springer, Cham, 2016.

[38] J. K. Lenstra and A. H. G. Kan, "Complexity of Vehicle Routing and Scheduling Problems", Networks (11:2), 1981, pp. 221-227. 